\title{
Diffusion MRI in spinal dural arterio-venous fistula: a case report
}

\author{
I Sibon*,1, P Ménégon ${ }^{2}, \mathrm{~N}$ Tafer $^{1}$ and V Dousset ${ }^{2}$ \\ ${ }^{1}$ Federation of Clinical Neurosciences, CHU Bordeaux, Bordeaux, France; ${ }^{2}$ Federation of Radiology, Department of \\ Neuroradiology, CHU Bordeaux, Bordeaux, France
}

\begin{abstract}
Study Design: Case report.
Objectives: Brain diffusion magnetic resonance imaging (MRI) and apparent diffusion coefficient (ADC) maps are useful to differentiate vasogenic and cytotoxic oedema during cerebovascular diseases. We investigate spinal cord diffusion MRI and ADC maps before and after treatment in one case of spinal dural arteriovenous fistula.

Setting: University of Bordeaux, France.

Methods: We used spinal cord diffusion MRI.

Results: We found a vasogenic oedema that disappears after treatment.

Conclusion: This case report confirms the interest of spinal cord diffusion and ADC maps to differentiate vasogenic and cytotoxic oedema at the spinal cord level.

Spinal Cord (2006) 44, 315-317. doi:10.1038/sj.sc.3101859; published online 25 October 2005
\end{abstract}

Keywords: diffusion; MRI; arterio-venous fistulae

\section{Introduction}

Spinal dural arterio-venous fistulas (SDAVF), while uncommon, are a cause of acute paraplegia. ${ }^{1}$ Magnetic resonance imaging (MRI) of the spinal cord should be the first radiological investigation of myelopathy. MRI abnormalities depicted during the investigation of SDAVF, such as signal hyperintensity in T2-weighted (T2-wi) images, enlargement of the cord and dilatation of the perimedullary veins, frequently disappear after treatment and are often correlated with clinical state. ${ }^{2,3}$ Whereas congestive myelopathy with vasogenic oedema is often associated with a good prognosis, cytotoxic oedema related to hypoxia and venous infarction often predicts a bad outcome. ${ }^{4}$ Several groups recently reported the usefulness of diffusion-weighted imaging (DWI) and apparent diffusion coefficient (ADC) maps to reveal early spinal cord infarction. ${ }^{5-7}$ The use of ADC maps may help to differentiate vasogenic from cytotoxic oedema in the spinal cord. We illustrate the usefulness of DWI and ADC maps for diagnosing and predicting the prognosis of a reversible acute myelopathy due to vasogenic oedema accompanying a SDAVF.

\section{Case report}

A 58-year-old man with a history of arterial hypertension, presented with an acute paraparesia. He reported a

*Correspondence: I Sibon, Research and Training Building, McGill University, Department of Psychiatry, 1033 Pine Avenue West, Montreal, QC, Canada H3A 1A1 2-month history of bilateral foot paresthesia, episodic sphincter dysfunction and, in the preceding week, recurrent episodes of intermittent paraparesia. Clinical examination revealed a mild paraparesia with hyporeflexia of the lower limbs and bilateral Babinski sign. Urinary retention was complete. All neurological symptoms spontaneously disappeared after 1 week.

Spinal cord MRI was performed $12 \mathrm{~h}$ after the clinical onset, with T1-weighted (T1-wi), T2-wi and DWI sequences. The T2-wi sequence showed high intramedullar signal intensity in the lower thoracic part of the spinal cord, and the conus terminalis combined with surrounded spinal cord enlargement and dilated perimedullary veins, suggesting SDAVF (Figure 1a). DWI showed an intramedullar highintensity signal of the corresponding area (Figure 1b). The ADC map, defined as a mean of three different ROIs, showed an increase in water self-diffusion in that area $\left(1.10^{3} \mathrm{~mm}^{2} / \mathrm{s}\right)$ while it was normal in the apparently unaffected upper thoracic spinal cord $\left(0.7 \times 10^{3} \mathrm{~mm}^{2} / \mathrm{s}\right)\left(\right.$ Ries et al) ${ }^{8}$ (Gyroscan NT). Conventional spinal angiography confirmed the diagnosis of SDAVF at the T12 spinal artery level (Figure 2). Endovascular treatment was rejected because of the complex anatomy of the arterio-venous malformation, so surgical clipping of the afferent feeder was performed. After 2 months the patient was free of any neurological symptoms and MRI T2-wi and DWI normalized $\left(0.710^{3} \mathrm{~mm}^{2} / \mathrm{s}\right.$ ) (Figure 1c) without spinal cord atrophy. 

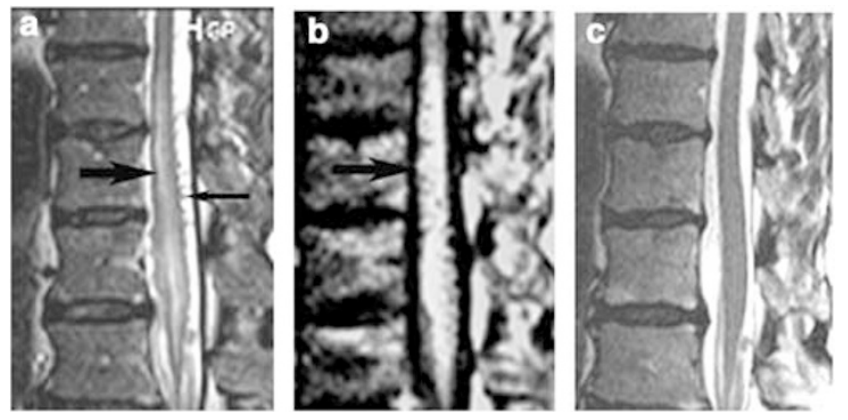

Figure 1 (a) Sagital T2-wi images of the spinal cord: High signal intensity (large arrow) and perimedullary voids of signal (thin arrow) in the lower thoracic part of the spinal cord, and the conus terminalis. (b) Diffusion MRI showing high signal intensity (large arrow) at the lower thoracic part of the spinal cord and the conus terminalis compared to the middle part of the thoracic cord. (c) T2-wi 2 months later showing a disappearance of the abnormalities

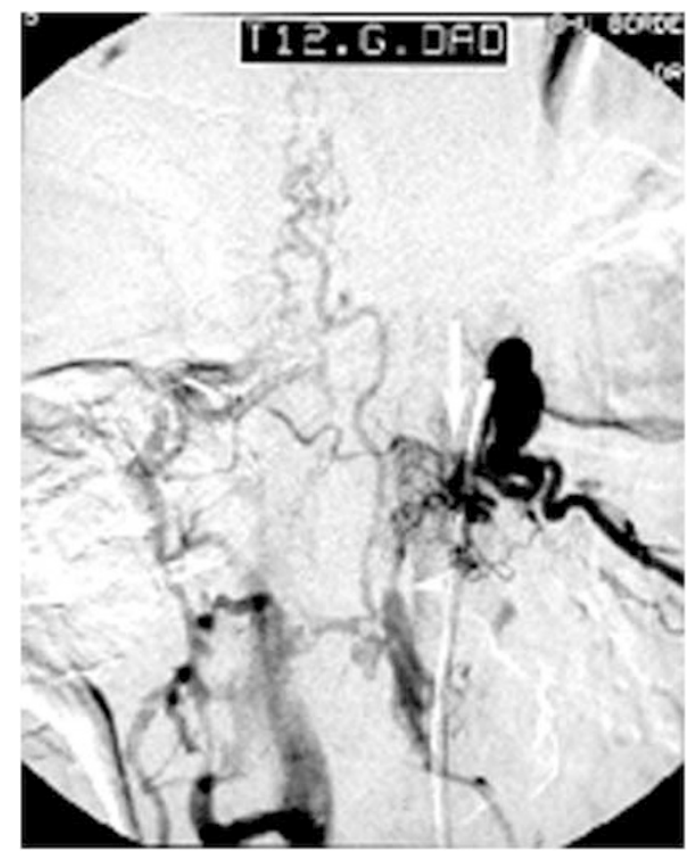

Figure 2 Subtracted anteroposterior spinal angiograms of left T12 intercostal artery showing the spinal dural fistula (arrow) and the spinal draining veins (arrowhead)

\section{Discussion}

MRI is the method of choice for the detection and diagnosis of many disorders in the spine because of its inherent sensitivity to soft tissues and its capability of displaying long segments of the vertebral column in one examination. Among the different MRI sequences available, DWI and measurement of the ADC may be a powerful method of detecting structural damage to the spinal cord. Experimental and more recently clinical data have suggested that DWI is more sensitive to spinal cord damage and recovery than conventional MRI and may also contribute to the pathophysiologic understanding of the underlying disorder.,, 10

DWI high signal images have been described with both, decreases and increases of the ADC. Increase of the ADC has been described in many conditions such as myelitis, multiple sclerosis and myelomalacia. ${ }^{8,10,11}$ This increase of the ADC could be related to the vasogenic oedma related to the inflammation associated with these diseases. Conversely, restriction of the ADC has mainly been described in ischaemic vascular diseases such as the acute phase of spinal cord infarct and some cases of spondylitic myelopathy. ${ }^{5-7,12}$ In these cases, the ADC restriction is currently attributed to cytotoxic oedema. We have recently shown the potential of the DWI technique in the diagnosis of acute spinal cord infarction using the same multishot echo planar imaging sequence which yields DWI images with B factors of 300 and $600 \mathrm{~s} / \mathrm{mm}^{2}$ in six directions which allows ADC and (diffusion) tensor maps to be obtained. ${ }^{7,9}$ The present case report suggests the ability of DWI to diagnose the mechanism of acute paraplegia during SDAVF. Acute paraplegia observed during SDAVF could be related to vasogenic oedema due to venous congestion or to spinal cord infarcts resulting from the thrombosis of the draining vein. ${ }^{1}$ DWI high signal intensity and increase in ADC, as observed in this case, suggest a vasogenic oedema. ${ }^{13}$ These changes may have resulted from increased blood volume in the spinal cord, as previously described with spinal perfusion MRI. ${ }^{14}$ Our data suggests that these sequences will likely play an important role in differentiating potentially reversible (vasogenic oedema due to venous congestion) from irreversible injury (cytotoxic oedema due to spinal cord venous infarct) during SDAVF. ${ }^{13}$ Thus, DWI and ADC could be useful tools to predict the prognosis before treatment of the SDAVF.

\section{References}

1 Caragine Jr LP, Halbach VV, Ng PP, Dowd CF. Vascular myelopathies-vascular malformations of the spinal cord: presentation and endovascular surgical management. Semin Neurol 2002; 22: 123-132.

2 Willinsky RA, terBrugge K, Montanera W, Mikulis D, Wallace MC. Post treatment MR findings in spinal dural arteriovenous malformations. AJNR Am J Neuroradiol 1995; 16: 2063-2071.

3 Rodesch G, Lasjaunias P. Spinal cord arteriovenous shunts: from imaging to management. Eur J Radiol 2003; 46: $221-232$.

4 Rodesch G, Berenstein A, Lajaunias P. Vasculature and vascular lesions in the spine and spinal cord. In: Manelfe $\mathrm{C}$ (ed). Imaging of the spinal cord (Chapter 17). Raven Press: New York 1992; 565: 98.

5 Sibon I, Menegon P, Moonen CT, Dousset V. Early diagnosis of spinal cord infarct using magnetic resonance diffusion imaging. Neurology 2003; 61: 1622.

6 Stepper F, Lovblad KO. Anterior spinal artery stroke demonstrated by echo-planar DWI. Eur Radiol 2001; 11: 2607-2610. 
7 Kuker W, Weller M, Klose U, Krapf H, Dichgans J, Nagele T. Diffusion-weighted MRI of spinal cord infarction - high resolution imaging and time course of diffusion abnormality. J Neurol 2004; 251: 818-824.

8 Ries M, Jones RA, Dousset V, Moonen CT. Diffusion tensor MRI of the spinal cord. Magn Reson Med 2000; 44: 884-892.

9 Quencer RM, Pattany PM. Diffusion-weighted imaging of the spinal cord: is there a future? Am J Neuroradiol 2000; 21: 1181-1182.

10 Demir A et al. Diffusion-weighted MR imaging with apparent diffusion coefficient and apparent diffusion tensor maps in cervical spondylotic myelopathy. Radiology 2003; 229: $37-43$.
11 Clark CA, Werring DJ, Miller DH. Diffusion imaging of the spinal cord in vivo: estimation of the principal diffusivities and application to multiple sclerosis. Magn Reson Med 2000; 43: 133-138.

12 Bammer $\mathrm{R}$ et al. Diffusion-weighted MR imaging of the spinal cord. AJNR Am J Neuroradiol 2000; 21: 587-591.

13 Sevick RJ et al. Cytotoxic brain edema: assessment with diffusion-weighted MR imaging. Radiology 1992; 185: 687-690.

14 Yanaka K, Matsumaru Y, Uemura K, Matsumura A, Anno I, Nose T. Perfusion-weighted MRI of spinal dural arteriovenous fistula. Neuroradiology 2003; 45: 744-747. 